\title{
OPINIÃO DE AGENTES COMUNITÁRIOS DE SAÚDE SOBRE A POLÍTICA ESPECÍFICA À SAÚDE MASCULINA
}

\author{
Rosineide Santana de Britoํㅡㄹ Danyelle Leonette Araújo dos Santos², Nathaly Ellen Maria Silva Ferreira ${ }^{3}$
}

\begin{abstract}
RESUMO: Pesquisa exploratória e descritiva, de abordagem quantitativa, cujo objetivo foi verificar a opinião dos Agentes Comunitários de Saúde que atuam na Estratégia Saúde da Família sobre a política de saúde específica para atender a população masculina. Participaram do estudo 64 Agentes Comunitários de Saúde, trabalhadores de quatro Unidades Básicas de Saúde, localizadas no Distrito Sanitário Oeste do Município de Natal - Rio Grande do Norte. Os dados foram coletados de julho a setembro de 2012 por meio de um formulário e tratados pela estatística descritiva. Verificou-se que, embora alguns participantes tenham revelado não conhecer a Política Nacional de Atenção Integral à Saúde do Homem, a maioria emitiu opiniões favoráveis acerca desta, fato justificado, principalmente, pelo seu caráter preventivo e educativo. O conhecimento evidenciado mostrou-se incipiente, mostrando a necessidade de a referida política ser propagada entre estes profissionais da atenção primária, visando sua concretização nos serviços de saúde.
\end{abstract}

DESCRITORES: Saúde do homem; Agentes comunitários de saúde; Programa Saúde da Família; Saúde pública.

\section{OPINIÓN DE AGENTES COMUNITARIOS DE SALUD SOBRE LA POLÍTICA ESPECÍFICA A LA SALUD MASCULINA}

RESUMEN: Investigación exploratoria y descriptiva, de abordaje cuantitativo, cuyo objetivo fue verificar la opinión de los Agentes Comunitarios de Salud que actúan en la Estrategia Salud de la Familia sobre la política de salud específica para atender a la población masculina. Participaron del estudio 64 Agentes Comunitarios de Salud, trabajadores de cuatro Unidades Básicas de Salud, ubicadas en el Distrito Oeste del Municipio de Natal - Rio Grande do Norte. Los datos fueron recogidos de julio a septiembre de 2012 por medio de formulario y sometidos a la estadística descriptiva. Se verificó que, mismo que algunos participantes tengan revelado no conocer la Política Nacional de Atención Integral a la Salud del Hombre, la mayoría tuvo opiniones favorables acerca de esta, hecho justificado, principalmente, por su carácter preventivo y educativo. El conocimiento evidenciado se mostró incipiente, indicando la necesidad de la referida política ser propagada entre estos profesionales de la atención primaria, a fin de concretarla en los servicios de salud. DESCRIPTORES: Salud del hombre; Agentes comunitarios de salud; Programa Salud de la Familia; Salud pública.

\section{THE OPINION OF COMMUNITY HEALTH WORKERS REGARDING THE POLICY SPECIFIC TO MALE HEALTH}

\begin{abstract}
This exploratory and descriptive research, with a quantitative approach, aimed to ascertain the opinion of Community Health Workers working in the Family Health Strategy regarding the health policy specific for attending the male population. 64 Community Health Workers participated in the study, who worked in four Primary Health Centers located in the West Health District of the city of Natal in the State of Rio Grande do Norte. The data were collected in July - September 2012 using a questionnaire and were treated using descriptive statistics. It was ascertained that, although some participants showed they did not know about the National Policy for Men's Integrated Healthcare, the majority expressed positive opinions about it, a fact mainly explained by its preventive and educational character. The knowledge evidenced was shown to be incipient, evidencing the need for the abovementioned policy to be publicized among these primary care professionals, with a view to its attainment in the health services. DESCRIPTORS: Men’s health; Community health workers; Family Health Program; Public health.
\end{abstract}

1Enfermeira. Doutora em Enfermagem. Professora da Universidade Federal do Rio Grande do Norte. Natal-RN-Brasil
2Enfermeira. Mestranda em Enfermagem. Bolsista CAPES. Universidade Federal do Rio Grande do Norte. Natal-RN-Brasil
${ }^{3}$ Acadêmica de Enfermagem. Bolsista REUNI de Iniciação Científica. Universidade Federal do Rio Grande do Norte. Natal-RN-Brasil

Autor correspondente:

Recebido: 01/03/2013

Danyelle Leonette Araújo dos Santos

Aprovado: 11/12/2013

Universidade Federal do Rio Grande do Norte

Rua Eugenópolis, 4807 - 59088-280 - Natal-RN-Brasil

E-mail: danyleonette@gmail.com 


\section{INTRODUÇÃO}

O Programa Saúde da Família foi elaborado em 1994, pelo Ministério da Saúde, com o objetivo de reorientar o modelo assistencial vigente no Brasil. No entanto, esse passou a ser considerado uma estratégia, por priorizar ações de promoção, prevenção e recuperação da saúde das famílias de forma contínua e integral. Assim, a Estratégia Saúde da Família (ESF) busca organizar a atenção primária de acordo com os princípios organizacionais e doutrinários do Sistema Único de Saúde (SUS) ${ }^{(1)}$.

Entretanto, para o alcance de tais princípios, a assistência prestada à comunidade é feita de forma multiprofissional. Deste modo, as equipes são compostas, minimamente, por um médico generalista, um enfermeiro generalista, um auxiliar de enfermagem e seis Agentes Comunitários de Saúde (ACS), podendo ainda contar com um dentista, um auxiliar de consultório dentário e um técnico em higiene bucal ${ }^{(2)}$.

Os ACS apresentam peculiaridades em seu processo de trabalho, merecendo destaque o fato de residirem na comunidade em que trabalham, conhecendo, portanto, as principais necessidades e anseios dos moradores. Isto possibilita a eles o reconhecimento dos determinantes saúde-doença, fato relevante para desenvolverem ações embasadas na realidade vivenciada pelos residentes na área de abrangência da $\operatorname{ESF}^{(3-4)}$.

Devido à proximidade dos ACS com a comunidade, a relação por eles estabelecida fundamenta-se em laços de confiança, os quais acarretam no surgimento de vínculos com a população. Estes, por sua vez, permitem que esses trabalhadores sejam reconhecidos como promotores de saúde nas diferentes atividades desenvolvidas. Nesse contexto, insere-se a visita domiciliária, a qual é considerada de suma importância na ESF, pois contribui para o levantamento de informações íntimas de cada família, inclusive de indivíduos que pouco frequentam o serviço de saúde, como é o caso dos homens.

Sobre esse assunto, diversos estudos vêm sendo desenvolvidos no âmbito da atenção primária em saúde sobre o público masculino, que, em geral, é negligenciado nas instituições de saúde. Isto porque, pelo fato de as Unidades Básicas de Saúde (UBS) promoverem ações destinadas a grupos populacionais, a escassez de programas assistenciais voltados aos homens gera lacunas no atendimento desses indivíduos ${ }^{(5-6)}$.

Esta realidade é um reflexo do estereótipo de gênero disseminado socialmente, o qual atribui aos homens características de força, poder, virilidade e invulnerabi- lidade. Tais adjetivos tendem a acarretar ônus à saúde desse público, visto a busca por assistência preventiva ser interpretada como um sinal de fraqueza masculina. Deste modo, os homens reprimem suas necessidades de saúde e protelam a busca por atendimento ${ }^{(7)}$, circunstância que os leva a um desfavorecimento nos indicadores de mortalidade.

Dentre as principais causas de mortes prematuras do grupo masculino estão aquelas desencadeadas por doenças crônico-degenerativas, como também por causas externas $^{(8)}$. Esta situação relaciona-se ao ideal de masculinidade adotado pelos homens, os quais, por sentirem-se invulneráveis, tendem a se expor a situações de perigo, tornando-se mais vulneráveis, gerando, assim, um paradoxo.

A situação desfavorável da saúde masculina impulsionou o Ministério da Saúde a elaborar, em 2008, a Política Nacional de Atenção Integral à Saúde do Homem (PNAISH). Esta tem o propósito de facilitar o acesso dos homens aos serviços de saúde e melhorar as suas condições de vida. A implementação desta política baseia-se em princípios e diretrizes, cujo objetivo é atender esse grupo populacional nos diferentes níveis de atenção, articulando-se com as demais políticas de saúde. Além disso, enfatiza a qualidade da assistência oferecida, reorganizando as ações, no intuito de transformar as UBS em espaços também masculinos ${ }^{(9)}$.

Contudo, o alcance dos referidos objetivos requer a capacitação e qualificação dos profissionais atuantes na ESF. Ademais, é imprescindível o empenho desses trabalhadores, pois o interesse deles apresenta-se essencial no desenvolvimento de estratégias voltadas ao público masculino, como também na concretização da PNAISH. Deste modo, considerando o fato de os trabalhadores da ESF serem os responsáveis pelo processo de cuidar, conhecer a opinião deles acerca da PNAISH é de suma importância para a consolidação desta política no âmbito da atenção primária. Nesse contexto, salienta-se a relevância dos ACS, pois, sua proximidade com os moradores da área de abrangência da ESF pode contribuir estimulando os homens a aderirem aos programas desenvolvidos pelas UBS, tornando-os sujeitos ativos na assistência em saúde.

Mediante as considerações expostas, questiona-se: qual a opinião dos ACS sobre a política de saúde específica para atender à população masculina? Assim sendo, o presente estudo objetivou verificar a opinião dos ACS que atuam na ESF sobre a política de saúde específica para atender a população masculina. 


\section{MÉTODO}

O presente estudo faz parte do projeto "Concepção de profissionais de saúde sobre a inserção de programas específicos para atender o homem na atenção primária à saúde". Trata-se de uma pesquisa exploratória, descritiva com abordagem quantitativa, desenvolvida no Distrito Sanitário Oeste do Município de Natal - Rio Grande do Norte, Brasil. Embora a cidade seja dividida em cinco regiões administrativas, optou-se pelo referido Distrito devido ao número expressivo de equipes da ESF. Deste modo, a seleção das UBS foi realizada por meio de sorteio aleatório simples, sendo selecionadas quatro UBS.

Participaram do estudo 64 ACS, os quais foram selecionados considerando como critérios de inclusão: atuar nas UBS selecionadas e residir na comunidade em que trabalhavam. Foram excluídos da amostra aqueles em férias ou de licença médica. Salienta-se o fato de apenas três ACS terem se recusado em participar do estudo.

A coleta de dados ocorreu no período de julho a setembro de 2012, utilizando-se um formulário constituído de questões abrangendo variáveis sociodemográficas e específicas ao objeto de estudo. Inicialmente, o possível participante foi contatado no intuito de ser esclarecido o objetivo da pesquisa, a fim de verificar sua disponibilidade em participar do estudo. Caso demonstrasse interesse, solicitava-se a assinatura do Termo de Consentimento Livre e Esclarecido, o qual ressaltava a garantia do seu anonimato, como também o uso de suas informações apenas para fins científicos, conforme preconiza a Resolução 466/12 $2^{(10)}$. As entrevistas ocorreram individualmente, em uma sala da própria UBS, a qual se encontrava disponível, isto é, sem realização de atendimentos à comunidade no instante da coleta de dados.

Ressalta-se que precedeu esta etapa a anuência do secretário de saúde municipal, autorização dos diretores das UBS selecionadas, bem como aprovação do Comitê de Ética em Pesquisa da Universidade Federal do Rio Grande do Norte, com parecer favorável n. 293/2011 e CAAE n. 0114.0.051.000-11.

Os dados coletados foram tabulados no programa Microsoft Word 2007, analisados pela estatística descritiva e apresentados em forma de tabelas. A discussão dos resultados teve como respaldo a PNAISH, bem como a literatura científica sobre atenção primária em saúde e ESF.

\section{RESULTADOS}

A partir dos dados obtidos, observa-se na Tabela 1 que 50(78\%) ACS eram do sexo feminino e $14(22 \%)$ do sexo masculino. Relativo à faixa etária, 20(31\%) encontravam-se com idade entre 36 e 40 anos, seguida de $16(25 \%)$ que referiram ter entre $41-45$ anos. Quanto ao estado conjugal, 35(55\%) indivíduos relataram estarem casados e 22(35\%) declararam-se solteiros. Sobre o grau de escolaridade, $48(75 \%)$ sujeitos informaram possuir o ensino médio completo e apenas $1(1 \%)$ declarou não ter finalizado os estudos. Tratando-se do tempo de atuação como ACS, constatou-se o fato de, na amostra estudada, não haver indivíduos trabalhando neste ofício por um período inferior a cinco anos.

Em relação ao conhecimento dos ACS acerca da PNAISH, a Tabela 2 mostra que 14(22\%) desconheciam a política e 50(78\%) revelaram possuir algum entendimento sobre ela, adquirido, em sua maioria, na UBS de atuação.

Quando questionados sobre a sua opinião a respeito da PNAISH, é importante destacar que o maior quan-

Tabela 1 -Características sociodemográficase tempodeatuaçãode Agentes ComunitáriosdeSaúde.Natal-RN-Brasil,2012

\begin{tabular}{|c|c|c|c|}
\hline Variável & & $\mathbf{n}$ & $\%$ \\
\hline \multicolumn{4}{|l|}{ Sexo } \\
\hline & Feminino & 50 & 78 \\
\hline & Masculino & 14 & 22 \\
\hline \multicolumn{4}{|l|}{ Idade } \\
\hline & $30-35$ anos & 15 & 23 \\
\hline & $36-40$ anos & 20 & 31 \\
\hline & $41-45$ anos & 16 & 25 \\
\hline & $46-50$ anos & 08 & 13 \\
\hline & $51-55$ anos & 04 & 06 \\
\hline & $56-60$ anos & 01 & 02 \\
\hline \multicolumn{4}{|c|}{ Estado Conjugal } \\
\hline & Casado & 35 & 55 \\
\hline & Solteiro & 22 & 35 \\
\hline & Divorciado & 06 & 09 \\
\hline & Viúvo & 01 & 01 \\
\hline & Total & 64 & 100 \\
\hline \multicolumn{4}{|c|}{ Escolaridade } \\
\hline & $\mathrm{EM}^{*}$ Incompleto & 01 & 01 \\
\hline & EM* Completo & 48 & 75 \\
\hline & ES** Incompleto & 14 & 23 \\
\hline & ES** Completo & 01 & 01 \\
\hline \multicolumn{4}{|c|}{ Tempo de atuação como ACS } \\
\hline & $1-5$ anos & 0 & 0 \\
\hline & $6-10$ anos & 20 & 31 \\
\hline & $11-15$ anos & 23 & 36 \\
\hline & $16-20$ anos & 21 & 33 \\
\hline Total & & 64 & 100 \\
\hline
\end{tabular}


titativo de respostas positivas voltou-se à existência de uma política específica à saúde do homem, mesmo entre aqueles que mencionaram desconhecê-la. Deste modo, conforme se observa na Tabela 3, a maioria considerou ser importante a existência da PNAISH.

De acordo com a Tabela 4, dentre as justificativas apresentadas sobre a opinião emitida, o caráter preventivo e educativo da PNAISH foi mencionada por 14

Tabela 2-Modo de obtenção do conhecimento sobre a Política Nacional de Atenção Integral à Saúde do Homem entre Agentes Comunitários de Saúde. Natal-RN-Brasil, 2012

\begin{tabular}{lcc}
\hline Variável & n & \% \\
\hline Unidade Básica de Saúde & 23 & 46 \\
Palestras com profissionais de saúde & 11 & 22 \\
Mídia & 16 & 32 \\
\hline Total & $\mathbf{5 0}$ & $\mathbf{1 0 0}$ \\
\hline
\end{tabular}

(22\%) ACS. Além desta, a possibilidade de estimular a procura de homens pelos serviços de saúde e de estimular os cuidados desses indivíduos com a saúde foram apontadas, respectivamente, por $12(19 \%)$ e $11(17 \%)$ participantes. Apesar de 16 (25\%) ACS não saberem informar as razões para considerarem a positividade da política, eles também emitiram opinião favorável à sua existência.

Tabela 3 - Importância atribuída por Agentes Comunitários de Saúde à Política Nacional de Atenção Integral à Saúde do Homem. Natal-RN-Brasil, 2012

\begin{tabular}{lcc}
\hline Variável & $\mathbf{n}$ & $\mathbf{\%}$ \\
\hline Boa & 18 & 28 \\
Muito boa & 04 & 6 \\
Ótima & 13 & 21 \\
Excelente & 01 & 1 \\
Importante & 24 & 38 \\
Não emitiu opinião & 04 & 6 \\
\hline Total & $\mathbf{6 4}$ & $\mathbf{1 0 0}$ \\
\hline
\end{tabular}

Tabela 4 - Justificativas emitidas pelos Agentes Comunitários de Saúde quanto à positividade da Política Nacional de Atenção Integral à Saúde do Homem. Natal-RN-Brasil, 2012

\begin{tabular}{llc}
\hline Justificativas & n & \% \\
\hline Possui caráter preventivo e educativo & 14 & 22 \\
Estimula a procura dos homens pelos serviços de saúde & 12 & 19 \\
Estimula os cuidados dos homens com a saúde & 11 & 17 \\
Aumenta a acessibilidade e amplia o atendimento ao homem & 07 & 11 \\
Aumenta o interesse dos profissionais pela saúde do homem & 03 & 5 \\
Promove atenção especializada à saúde do homem & 01 & 1 \\
Não soube informar & 16 & 25 \\
\hline Total & $\mathbf{6 4}$ & $\mathbf{1 0 0}$ \\
\hline
\end{tabular}

\section{DISCUSSÃO}

A análise dos dados mostrou predominância do sexo feminino entre os pesquisados, o que se explica pela forte presença de mulheres no setor saúde. Isto guarda relação com o estereótipo feminino arraigado culturalmente, no qual se atribui a mulher adjetivos como docilidade, delicadeza e paciência, características fundamentais na realização do cuidado ${ }^{(11-12)}$. Apesar de estas características serem tidas como relevantes para profissões cujo enfoque é a assistência ao outro - como é o caso da categoria profissional dos ACS -, entende-se que este maior contingente feminino nas UBS estabelece barreiras e inibe a verbalização masculina de seus problemas de saúde. Além disso, dificulta a sensação nos homens de sentirem-se pertencentes aos serviços de atenção primária ${ }^{(5,7)}$.
Relativo à idade dos participantes, observou-se maior número de ACS inseridos na faixa etária entre 36 e 40 anos. Este achado condiz com a idade produtiva para adultos no Brasil, cujo início se dá aos 15 anos e o término aos 59 anos. Ademais, corrobora aos resultados de outro estudo cuja faixa etária predominante dos ACS foi de 30-39 anos $^{(13)}$. Quanto ao estado conjugal, verificou-se maior quantitativo de ACS casados, realidade semelhante à encontrada em outras pesquisas realizadas junto a esses profissionais ${ }^{(14-15)}$.

Com relação ao grau de escolaridade, este se apresentou maior do que o exigido pela Lei n. 11.350, que estabelece o ensino fundamental completo para exercer a função de $\mathrm{ACS}^{(16)}$. Este fato também foi observado em outros estudos, os quais identificaram esta situação como importante para qualificação destes profissionais ${ }^{(13,15)}$. Neste contexto, é válido salientar o percentual 
expressivo da amostra estudada que declarou cursar o ensino superior. Tal fato pode ser associado às atuais condições de acesso às Universidades, promovidas pelo governo federal, por meio de programas de auxílio à educação, os quais facilitam o ingresso das pessoas ao ensino superior.

Tratando-se do tempo médio de atuação como ACS, constatou-se período expressivo de participantes neste cargo, aspecto considerado positivo para a comunidade. Esta positividade relaciona-se a um menor rodízio desses trabalhadores e, consequentemente, maior estabelecimento de vínculos junto à população. Para o trabalho dos ACS, a aproximação com as pessoas residentes na área da ESF é fundamental no desenvolvimento de suas atividades.

Quanto ao conhecimento dos ACS sobre a PNAISH, 50(78\%) revelaram possuir algum entendimento sobre ela. E, dentre estes, 23(46\%) adquiriram tal conhecimento na UBS onde trabalham e $11(22 \%)$ mencionaram ter obtido esta informação em palestras com profissionais de saúde. Esses resultados são relevantes, pois levam a crer na existência de discussões sobre políticas de saúde entre os trabalhadores da ESF. Reconhece-se que os espaços das UBS apresentam-se como apropriados na promoção de estratégias de Educação Permanente em Saúde aos profissionais. Essa consiste em uma forma de estabelecer transformações no processo de trabalho, qualificando os serviços de saúde e expandindo o acesso a estes. Sua implantação contribui para melhorias no desempenho dos trabalhadores da saúde, pois os capacitam para fazê-los lidarem com as diferentes conjunturas do cenário de saúde ${ }^{(17)}$.

A mídia foi outra forma de aquisição de conhecimento sobre a PNAISH, mencionada por $16(32 \%)$ dos ACS, evidenciando o papel exercido pelos meios de comunicação quanto ao repasse de informações sobre políticas e estratégias em saúde. Ademais, o fato de uma quantidade expressiva destes trabalhadores ter obtido tal conhecimento apenas por veículos midiáticos permite evidenciar, ainda, o caráter verticalizado das ações neste campo do saber, o que pode dificultar a implantação e concretização de novas políticas públicas.

Embora 14(22\%) participantes tenham informado não possuir conhecimento acerca da PNAISH é importante destacar que todos emitiram opinião sobre a existência de uma política de saúde voltada ao público masculino. Assim sendo, a maioria dos ACS considerou a política como benéfica à saúde dos homens. Este achado apresenta-se relevante, pois a opinião favorável destes profissionais contribui para maior aceitação de uma nova política, sobretudo quando esta é específica a um grupo populacional com o qual não estão acostumados a lidar em sua rotina de trabalho.

Ao reconhecerem como positiva a existência da PNAISH, diversas justificativas foram emitidas, destaca-se o caráter preventivo e a possibilidade de facilitar o acesso masculino aos serviços de saúde apresentaram-se como as principais razões para a PNAISH ser considerada benéfica. Particularizando o caráter preventivo, reconhece-se que desenvolver estratégias de prevenção voltada aos homens é desafiador. Tal concepção ancora-se, especialmente, nos padrões culturais, capazes de impor barreiras para estes indivíduos buscarem as UBS antes de eles apresentarem sintomas denotativos de alguma doença estabelecida ${ }^{(18)}$.

Outras justificativas acerca da positividade da PNAISH foram mencionadas pelos ACS, quais sejam: estímulo aos homens buscarem os serviços de atenção primária; incentivo para o grupo masculino cuidar de si; aumento da acessibilidade, como também ampliação do atendimento ao público masculino nos serviços e do interesse dos profissionais pela saúde do homem. Acredita-se que estas respostas estejam relacionadas ao conhecimento - ainda que incipiente -, adquirido pelos ACS acerca do Programa, pois as justificativas mencionadas guardam relação com os objetivos e princípios da política.

No âmbito desses princípios é necessário estimular a ida do público masculino às instituições primárias de saúde, como também promover o acesso desse grupo aos serviços de saúde de maneira hierarquizada e em rede. Para isto, é imperativo envolver os homens nas ações desenvolvidas nos serviços de saúde com vistas a eles apreenderem tais espaços também como masculinos ${ }^{(9)}$.

Contudo, a justificativa dos benefícios da PNAISH apontada por $1 \%$ dos ACS quanto à possibilidade de uma atenção especializada no campo da saúde masculina, apresenta-se em desacordo com o preconizado pelo Ministério da Saúde ${ }^{(9)}$. Pois, esse conceber a entrada dos homens no sistema de saúde, por meio da atenção especializada reforça a ideia de estes indivíduos permanecerem à margem das ações preventivas, desenvolvidas no contexto da atenção primária.

Outro aspecto que merece ser analisado diz respeito ao fato de $16(25 \%)$ ACS não terem apontado razões para conceberem a PNAISH como benéfica. Isto, possivelmente, vincula-se ao desconhecimento de alguns sujeitos acerca dos propósitos da política voltada à saúde do homem, demonstrando a necessidade de esta ser amplamente propagada nos setores de atenção primária. 


\section{CONCLUSÕES}

Os resultados obtidos com este estudo possibilitaram responder ao questionamento de pesquisa. Assim, os dados mostram que apesar do conhecimento incipiente acerca dos propósitos da PNAISH, os ACS possuem opinião favorável sobre esta política. Tal positividade, ancorada em aspectos voltados à prevenção de agravos à saúde masculina, vincula-se ao entendimento prévio dos ACS sobre os princípios e diretrizes do SUS, os quais devem ser considerados em políticas voltadas à saúde da população.

Diante disto, evidencia-se a necessidade de a PNAISH ser mais bem discutida entre gestores e profissionais da atenção primária, com vistas a sua concretização nos serviços de saúde. Nesse sentido, esses trabalhadores são fundamentais, sobretudo, por suas características peculiares dentro da equipe da ESF, as quais podem ser consideradas na divulgação da referida política junto aos usuários do sexo masculino. A partir disto, espera-se que estes trabalhadores possam estimular os homens a buscarem os serviços de saúde, no intuito de minimizar riscos e precaver agravos passíveis de prevenção.

\section{REFERÊNCIAS}

1. Giovanella L, Mendonça MHM, Almeida PF, Escorel S, Senna MCM, Fausto MCR, et al. Saúde da família: limites e possibilidades para uma abordagem integral de atenção primária à saúde no Brasil. Ciênc. saúde colet. 2009;14(3):783-94.

2. Ministério da Saúde (BR). Portaria n. 1886/GM, de 18 de dezembro de 1997. Aprova as Normas e Diretrizes do programa de Agentes Comunitários de Saúde e do Programa de Saúde da Família. [Internet] [acesso em 22 jan 2013]. Disponível: http://189.28.128.100/dab/docs/ legislacao/portaria1886_18_12_97.pdf.

3. Bachilli RG, Scavassa AJ, Spiri WC. A identidade do agente comunitário de saúde: uma abordagem fenomenológica. Ciênc. saúde colet. 2008;13(1):51-60.

4. Santana JCB, Vasconcelos AL, Martins CV, Barros JV, Soares JM, Dutra BS. Agente Comunitário de Saúde: percepções na estratégia saúde da família. Cogitare enferm. [Internet] 2009;14(4) [acesso em 21 jan 2013]. Disponível: http://ojs.c3sl.ufpr.br/ojs2/index.php/ cogitare/article/view/16377/10858.

5. Brito RS, Santos DLA, Maciel PSO. Olhar masculino acerca do atendimento na Estratégia Saúde da Família.
Rev. Rene. [Internet] 2010;11(4) [acesso em 20 jan 2013]. Disponível: http://www.revistarene.ufc.br/vol11n4_pdf/ a15v11n4.pdf.

6. Figueiredo WS, Schraiber LB. Concepções de gênero de homens usuários e profissionais de saúde de serviços de atenção primária e os possíveis impactos na saúde da população masculina. Ciênc. saúde colet. 2011;16(Suppl 1):935-44.

7. Gomes R. Sexualidade masculina, gênero e saúde. Rio de Janeiro: Fiocruz; 2008.

8. Stevens A, Schmidt MI, Duncan BB. Gender inequalities in non communicable disease mortality in Brazil. Ciênc. saúde colet. 2012;17(10):2627-34.

9. Ministério da Saúde (BR). Política Nacional de Atenção Integral à Saúde do Homem: Princípios e Diretrizes. Brasília: Ministério da Saúde; 2008.

10. Ministério da Saúde (BR). Conselho Nacional de Saúde. Diretrizes e normas regulamentadoras de pesquisa envolvendo seres humanos. Resolução n. 466, de 12 de dezembro de 2012. Brasília; 2012.

11. Cecilio LCO. Gênero e estudos organizacionais: apontamentos para futuros estudos. Ciênc. saúde colet. 2009;14(4):107-8.

12. Barbosa RHS, Menezes CAF, David HMSL, Bornstein VJ. Gênero e trabalho em saúde: um olhar crítico sobre o trabalho de agentes comunitárias/os de saúde. Interface - Comunic., Saude, Educ. 2012;16(42):751-65.

13. Santos KT, Saliba NA, Moimaz SAS, Arcieri RM, Carvalho ML. Agente Comunitário de Saúde: perfil adequado à realidade do Programa Saúde da Família? Ciênc. saúde colet. 2011;16(Suppl 1):1023-8.

14. Cozer TB, Miotto MHMB, Pandolfi M. Perfil do Agente Comunitário de Saúde de Colatina, Espírito Santo. Rev. Odontol. [Internet] 2008;10(3) [acesso em 28 jan 2013]. Disponível: http://periodicos.ufes.br/RBPS/article/ view/466/330

15. Silva ERP, Cazola LHO, Cheade MFM, Pícoli RP. Atuação dos Agentes Comunitários de Saúde na Estratégia Saúde da Família. Cogitare enferm. [Internet] 2012;17(4) [acesso em 20 jan 2013]. Disponível: http://ojs.c3sl.ufpr.br/ojs2/index.php/cogitare/article/ view/30359/19636.

16. Brasil. Lei n. 11.350, de 5 de outubro de 2006. Regulamenta o $\S 5^{\circ}$ do art. 198 da Constituição, dispõe 
sobre o aproveitamento de pessoal amparado pelo parágrafo único do art. $2^{\circ}$ da Emenda Constitucional $\mathrm{n}^{\circ}$ 51, de 14 de fevereiro de 2006, e dá outras providências. Diário Oficial da República Federativa do Brasil, Brasília, 06 out. 2006. Seção 1.

17. Sarreta FO. Educação Permanente em Saúde para os trabalhadores do SUS [Internet]. São Paulo: Editora UNESP; 2009 [acesso em 23 jan 2013]. Disponível: http://books.scielo.org/id/29k48

18. Knauth DR, Couto MT, Figueiredo WS. A visão dos profissionais sobre a presença e as demandas dos homens nos serviços de saúde: perspectivas para a análise da implantação da Política Nacional de Atenção Integral à Saúde do Homem. Ciênc. saúde colet. 2012;14(10):2617-26. 